\title{
MOLECULES IN THE ENVELOPES OF LATE-TYPE STARS
}

\author{
R. LUCAS \\ IRAM \\ 300 rue de la Piscine \\ 38406 Saint-Martin-d'Hères \\ FRANCE
}

\section{Introduction}

With physical conditions very similar to those of molecular clouds, circumstellar envelopes (CSEs) are the sites for a rich chemistry. Hovever their simpler geometries, the variations in elemental composition, and the short time scales of the outflows, make them objects of particular interest to the astrochemist. For a recent and general review of circumstellar chemistry the reader may refer to Omont (1990). Here we give only a very short overview (Section 2), then we report on some of the very recent observational advances in this field (Section 3).

\section{Overview of circumstellar chemistry}

LTE is expected to be valid in the photosphere and in the very inner layers, where the temperature and the density are high. Calculations by Tsuji (1987) and others, show most radicals such as $\mathrm{CN}, \mathrm{HNC}, \mathrm{NH}_{3}$, cyanopolyynes (except $\mathrm{HC}_{3} \mathrm{~N}$ ) cannot be formed in LTE. Outside of the LTE region, radical reactions are dominated by reactions by $H$, the abundance of which is highly uncertain. Grain processes are also important in this region. Ice has been found on grains in some massive CSEs. Diffuse IR bands carriers (PAHs ?) are abundant in PPNs, but whether they are also important in the earlier phases is unknown (Frenklach and Feigelson 1989). Shocks are also probably present, related to pulsations and high velocity winds, which could dissociate abundant molecules, and result in the synthesis of otherwise unexpected products (HCN, CS in 0-rich CSEs).

The chemistry in the outer layers is dominated by photon induced processes. $\mathrm{CO}$ photodissociation is fast, but self shielding occurs. It is expected to be effective between $310^{16}$ and $10^{18} \mathrm{~cm}$, depending on the mass loss rate (Mamon et al. 1987). It leads to $\mathrm{C}$ and $\mathrm{C}^{+}$. Similar photodissociation chains starts from $\mathrm{C}_{2} \mathrm{H}_{2}$ and $\mathrm{HCN}$, in C-rich objects. Ionization is initiated by cosmic rays and by interstellar UV. The most abundant ions are $\mathrm{C}_{2} \mathrm{H}_{2}+, \mathrm{H}_{3}^{+}$, $\mathrm{H}_{3} \mathrm{O}^{+}, \mathrm{HCO}^{+}$, depending on the $\mathrm{C} / \mathrm{O}$ ratio. Chromospheric $\mathrm{UV}$ may also play a role in some objects, such as $\alpha$ Ori, or PNs, where $\mathrm{HCO}^{+}$is found in abundance. Ion-molecule reactions were proposed by Glassgold and Huggins (1985). They lead to the synthesis of 
$\mathrm{C}_{3} \mathrm{H}, \mathrm{C}_{3}, \mathrm{C}_{4} \mathrm{H}, \mathrm{HC}_{3} \mathrm{~N}, \mathrm{HNC}, \mathrm{SiC}_{2}, \mathrm{C}_{2} \mathrm{~S}, \mathrm{C}_{3} \mathrm{~S}, \ldots$ Radical reactions are also important due to abundance of radicals produced by photodissociation. They could synthesize $\mathrm{HC}_{3} \mathrm{~N}$, $\mathrm{C}_{4} \mathrm{H}, \mathrm{C}_{6} \mathrm{H}, \mathrm{SiC}_{4}, \mathrm{SO}, \mathrm{SO}_{2} \ldots$

\section{Recent observations}

\subsection{New Molecules}

Since the review by Lucas and Guélin (1990), several new molecules have been found in the last few years, all of them in the C-rich object IRC+10216:

- CP (Guélin et al. 1990). Its column density is $\simeq 10^{13} \mathrm{~cm}^{-2}$. Thermodynamics would imply that CP should be less abundant than PN, and much less than HCP, which is not found, while photochemistry would produce CP in the outer envelope. However from the profile shape, this molecule has an extent of $\simeq 15^{\prime \prime}$, which is smaller than that of photodissociation products.

- $\mathrm{H}_{2} \mathrm{CCC}$ (propadienylidene) was tentatively detected in $\mathrm{IRC}+10216$ with a column density of $\simeq 2.610^{12} \mathrm{~cm}^{-2}$ (Cernicharo et al. 1991a). It is the linear isomer of the $\mathrm{C}_{3} \mathrm{H}_{2}$ ring. - $\mathrm{H}_{2}$ CCCC (butatrienylidene), isomer of diacetylene, has been identified in IRC+10216 (Cernicharo et al. 1991b). The U-shaped lines imply a spatial distribution similar to that of $\mathrm{C}_{4} \mathrm{H}$. Formation by $\mathrm{C}_{2} \mathrm{H}_{2}{ }^{+}+\mathrm{C}_{2} \mathrm{H}_{2} \rightarrow \mathrm{C}_{4} \mathrm{H}_{3}{ }^{+}+\mathrm{H}$, followed by dissociative recombination, is proposed (Glassgold et al. 1987).

- The HCCN radical was found in the outer envelope with a column density $\simeq$ $1.210^{13} \mathrm{~cm}^{-2}$ (Guélin and Cernicharo 1991a). Its formation mechanism remains unclear.

- SiN (see Turner 1991)

\subsection{High-Resolution Observations of IRC +10216}

The nearby object IRC+10216 is by far the best studied of carbon-rich CSEs. Its chemical structure has been studied with the Hat Creek interferometer (Bieging and Rieu 1989). With more sensitive instruments it is now possible to obtain very detailed information on this prototype object.

The $30 \mathrm{~m}$ telescope has been used by Kahane et al. (1991) to study the distribution of $\mathrm{SiO}, \mathrm{SiS}, \mathrm{C}_{4} \mathrm{H}, \mathrm{HC}_{3} \mathrm{~N}$ and $\mathrm{SiC}_{2}$, in several transitions. While $\mathrm{SiO}$ and $\mathrm{SiS}$ are clearly present in the central regions $\left(\mathrm{r}<15^{\prime \prime}\right), \mathrm{C}_{4} \mathrm{H}$ is distributed in a hollow shell $\left(14^{\prime \prime}<r<26^{\prime \prime}\right)$. The case of $\mathrm{HC}_{3} \mathrm{~N}$ is less clear, since its $2 \mathrm{~mm}$ emission seems to fill a sphere, while at $3 \mathrm{~mm}$ Bieging and Rieu (1988) found it in a hollow shell. Finally the distribution of $\mathrm{SiC}_{2}$ emission is asymmetric and stronger in the southern part of the source.

With the Plateau de Bure interferometer, Guélin et al. (1991b) have observed $\mathrm{C}_{2} \mathrm{H}$ with high resolution and sensitivity. The $\mathrm{C}_{2} \mathrm{H}$ shell appears asymmetric (fig. 1). They simultaneously mapped emission from $\mathrm{C}_{5} \mathrm{H}$ and $\mathrm{C}_{4} \mathrm{H}$ in the $\nu_{7}$ vibrational state, which are both distributed in hollow shells. With the same instrument, Cernicharo et al. (1991c) mapped the emission of $\mathrm{NaCl}$ and of $\mathrm{SiC}_{2}$ (presented in a poster paper).

\subsection{Interferometric Observations of Thermal SiO in Evolved Stars}

Lucas et al. 1991, using the IRAM interferometer on Plateau de Bure, have observed the SiO $v=0 J=2-1$ emission from 12 evolved stars, mostly O-rich. Simple source models were 


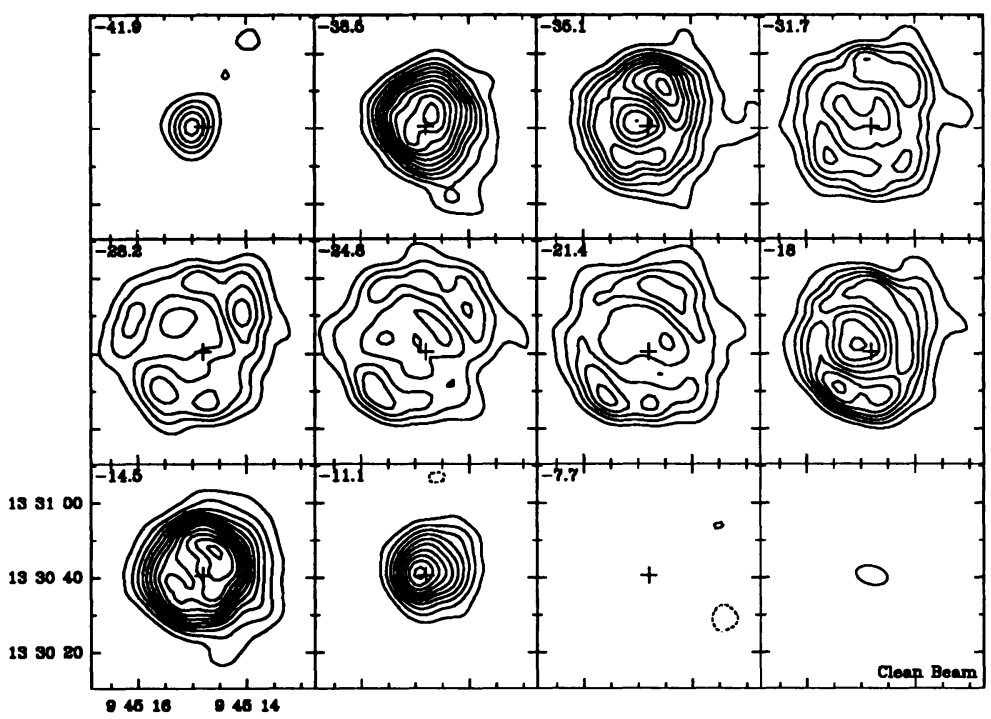

Figure 1: Combined 30-m and Plateau de Bure observations of $\mathrm{CCH}(\mathrm{N}, \mathrm{J})=(1,3 / 2)-(0,1 / 2)(87.3$ GHz) emission from IRC+10216. Channel maps labelled by LSR velocity in $\mathrm{km} \mathrm{s}^{-1}$. The resolution is 8.6" by 5.1", the contour spacing is $0.1 \mathrm{Jy}$ (Guélin et al. 1991b).

fitted in the visibility curves, thus determining fluxes and sizes. In some stars, the $u, v$ coverage was good enough to obtain maps.

In RX Boo, R Cas, $\chi$ Cyg, and IK Tau, the emission is circularly symmetric and centered on the star's optical position. For the other objects the results are also compatible with that geometry. The half intensity sizes are $0.9^{\prime \prime}$ to $2.4^{\prime \prime}$. They are larger than those expected from previous work, though much smaller than the extents of CO (Bujarrabal and Alcolea 1991). SiO is widespread within the envelopes up to $\sim 1-710^{15} \mathrm{~cm}$, and even further for the supergiants IRC+10420 and NML Cyg. The profile shapes are mostly gaussian, which indicates the emitting regions do not have the expected high and constant expansion velocity. This is confirmed by the distribution of size as a function of velocity, which shows no clear increase at the line center. Grain formation is proposed to continue in 0 -rich and S-type Miras as far as $5 \mathbf{1 0}^{15} \mathrm{~cm}$ from the central star, which would explain the large $\mathrm{SiO}$ abundance and extended acceleration region that are probably at the origin of the non-standard line profiles.

\subsection{Nitrogen Chemistry in O-rich Envelopes}

Olofsson et al. (1991) observed CO, ${ }^{13} \mathrm{CO}, \mathrm{CN}, \mathrm{HCN}$ and CS in TX Cam with the 30-m telescope. $\mathrm{HC}_{3} \mathrm{~N}, \mathrm{SiS}$, and $\mathrm{HNC}$ had been found by Lindqvist et al. 1988, but neither $\mathrm{OH}$ nor $\mathrm{H}_{2} \mathrm{O}$ masers are found. For $\mathrm{CN}$, and $\mathrm{CS}$, the observed abundances agree with theoretical models (Nejad and Millar 1988, Nercessian et al. 1989), but there is a strong disagreement for HCN. The low CN/HCN ratio seems particular to O-rich environments.

S. Guilloteau et al. (1991) have used the IRAM interferometer on Plateau de Bure, to observe the HCN $v=0 \quad J=1-0$ emission from 0 -rich evolved stars. They found that 
the extent of HCN emission, a few $10^{16} \mathrm{~cm}$, is comparable to that of the $1612 \mathrm{MHz} \mathrm{OH}$ masers. These sizes are in agreement with the assumption that HCN is formed by gas-phase reactions initiated by the photodissociation of $\mathrm{CH}_{4}$, as proposed by Nejad and Millar (1988) and Nercessian et al. (1989).

\subsection{Observations of Sulfur-bearing Species in Oxygen-rich EnVelopes}

$\mathrm{H}_{2} \mathrm{~S}$ was first detected in $\mathrm{OH231.8}+4.2$ by Ukita and Morris (1983), while $\mathrm{SO}_{2}$ and SO observed were found with the $30 \mathrm{~m}$ (Lucas et al. 1986, Guilloteau et al. 1986). A systematic study of $\mathrm{SO}_{2}, \mathrm{SO}, \mathrm{H}_{2} \mathrm{~S}$ has been undertaken with the $30 \mathrm{~m}$ (Omont et al. 1991). $\mathrm{H}_{2} \mathrm{~S}$ was detected in 15 O-rich stars in its ortho and para states. The line profiles of $\mathrm{H}_{2} \mathrm{~S}$ are much narrower than those of $\mathrm{SO}_{2}$, which indicates that $\mathrm{H}_{2} \mathrm{~S}$ is present in the inner regions (where acceleration probably occurs), while $\mathrm{SO}_{2}$ (and probably $\mathrm{SO}$ ) are in an outer shell. Modelization of the $\mathrm{H}_{2} \mathrm{~S}$ emission shows it comes from a region of radius $\simeq 10^{16} \mathrm{~cm} . \mathrm{SO}_{2}$ and $\mathrm{SO}$ are formed by reactions of $\mathrm{S}$ with $\mathrm{OH}$, in the $\mathrm{H}_{2} \mathrm{O}$ photodissociation region (a few $\left.10^{16} \mathrm{~cm}\right)$.

\subsection{Studies of CO EMIssion}

CO, detected in hundreths of CSEs, has been extensively used to derive mass losses, following Knapp and Morris (1985). Margulis et al. (1990) observed all M giants, S stars, and $O$ stars in the $2 \mu \mathrm{m}$ Sky Survey, with $\delta>10^{\circ}$. The line profiles are simple for $\mathrm{C}$ ans $S$ stars, but for $M$ stars they indicate complex envelope structures. Heske et al. (1990) found a deficiency of $\mathrm{CO}$ in $13 \mathrm{OH} / \mathrm{IR}$ stars, particularly in the most massive ones. The $(2-1) /(1-0)$ ratio increases drastically with increasing optical depth of the dust $(9.7 \mu \mathrm{m}$ feature turning into absorption). This may be due to low kinetic temperature, or to lower mass loss in the outer shells.

Olofsson et al. (1990) haved mapped carbon stars with detached envelopes (R Scl, U Ant, S Sct, TT Cyg). These are thin envelopes, detached from the stars (inner radii $>10^{17} \mathrm{~cm}$ ), which probably present episodic mass loss, triggered by helium shell flashes. Bujarrabal and Alcolea (1991) have mapped with the 30m telescope, seven O-rich stars (RR Aql, RX Boo, R Cas, S CrB, R Leo R LMi, and IK Tau), 1 S star ( $\chi$ Cyg), and 2 C stars (S Cep and V Cyg). Most objects are resolved, roughly circular in shape, and much smaller than the sizes predicted by envelope models, such as Knapp and Morris (1985).

Sahai (1990) developed a self-consistent model for the CO emission, including thermal equilibrium, and showed that mass loss derivation is very sensitive to the kinetic temperature law. In particular the gas may be cooled to temperatures below $2.7 \mathrm{~K}$ by adiabatic expansion, producing $\mathrm{CO} \mathrm{J}=1-0$ profiles with reduced intensity and width. This model was applied to the C-rich object U Cam. Truong-Bach et al. (1990) have observed and modelled the CO emission of CRL2688. In their two-shell model of IRC+10216 (1991), the mass loss was higher in the outer envelope $\left(410^{-5} \mathrm{M}_{\odot} / \mathrm{yr}\right)$ than in the inner $4^{\prime \prime}\left(2.510^{-5}\right.$ $\left.\mathbf{M}_{\odot} / \mathbf{y r}\right)$.

\subsection{The $200 \mathrm{KM} \mathrm{s}^{-1}$ Bipolar Outrlow in CRL618}

This outflow, discovered by Cernicharo et al. (1989) has been observed in HCN J=1-0 with the Plateau de Bure interferometer (Neri et al. 1991). The angular resolution was 2.4". The circumstellar envelope itself is extended, and centered on the radio continuum source. 
The red-shifted outflow emission comes from a an unresolved region $1^{\prime \prime}$ to the west of the HII region, while the blue-shifted high-velocity gas arises from a source af size $\simeq 2^{\prime \prime}$, partly in front of the HII region. The high velocity HCN is proposed to be formed behind shocks at the wind-envelope interface surface.

\section{References}

Bieging, J.H., N-Q-Rieu 1988, ApJ 329, L107

Bieging, J.H., Nguyen-Q.-Rieu 1990, in "From Miras to Planetary Nebulae: which Path for Stellar Evolution?", Eds M.O. Mennessier and A. Omont, p164

Bujarrabal, V., Alcolea, J. 1991, A\&A, to be published

Cernicharo, J., Guelin, M., Martin-Pintado, J., Peñalver, J., Mauersberger, R. 1989, A\&A 222, L1

Cernicharo, J., Gottlieb, C.A., Guélin, M., Killian, T.C., Paubert, G., Thaddeus, P., Vrtilek, J.M. 1991a, ApJ 368, L39

Cernicharo, J., Gottlieb, C.A., Guelin, M., Killian, T.C., Thaddeus, P., Vrtilek, J.M. 1991b, ApJ 368, L43

Cernicharo, J., Guélin, M., Lucas, R., 1991c, in preparation

Frenklach, M., Feigelson, E.D. 1989, ApJ 341, 372

Glassgold, A.E., Mamon, G.A., Omont, A., Lucas, R. 1987, A\&A 180, 183

Guélin, M., Cernicharo, J., Paubert, G., Turner, B.E. 1990, A\&A 230, L9

Guélin, M, Cernicharo, J. 1991a, A\&A 244, L21

Guélin, M., Cernicharo, J., Guilloteau, S., Lucas, R., 1991b, in preparation

Guilloteau., S., Lucas, R., N-Q-Rieu, Omont, A. 1986 A\&A 165, L1

Guilloteau, S., Forveille, T., Lucas, R., Morris, M., Radford, S.J.E., Fuente, A. 1991, in preparation

Glassgold, A.E., Huggins, P.J. 1985, M-Type stars, ed. by H.R. Johnson and F. Querci (NSF-CNRS 1988), p291

Heske, A., Forveille, T., Omont, A., van der Veen, W.E.C.J., Habing, H. 1990, A\&A 239, 173

Jackson, J.M., N-Q-Rieu 1988, ApJ 335, L83

Kahane, C. et al. 1991, paper presented to the IAU.

Knapp, G.R., Morris, M. 1985, ApJ 292, 640

Lindqvist, M., Nyman, L.-A., Olofsson, H., Winnberg, A., 1988, A\&A 205, L15

Lucas, R., Omont, A., Guilloteau, S., N-Q-Rieu, 1986, A\&A 154, L12

Lucas, R., and Guélin, M. 1990, in "Submillimetre Astronomy", G.D. Watt and A.S.Webster eds., p97

Lucas R., Bujarrabal, V., Guilloteau, S., Bachiller, R., Baudry, A., Cernicharo, J., Delannoy, J., Forveille, T., Guélin, M., Radford, S.J.E., 1991, submitted to A\&A

Mamon, G.A., Glassgold, A.E., Huggins, P.J. 1988, ApJ 328, 797

Margulis, M., Van Blerkom, D.J., Snell, R.L., Kleinmann, S.G. 1990, ApJ 361, 673

Nejad, L.A.M., Millar, T.J. 1988, MNRAS 230, 79

Nercessian, E., Guilloteau, S., Omont, A., Benayoun, J.J. 1989, A\&A 210, 225

Neri, R., Garcia-Burillo, S., Cernicharo, J., Guélin, M., Guilloteau, S., and Lucas, R. 1991, in preparation

Olofsson, H., Carlström, U., Eriksson, K., Gustafsson, B., Willson, L.A. 1990, A\&A 230, L13

Olofsson, H., Linsqvist, M., Nyman, L.-A., Winnberg, A., N-Q-Rieu, 1991, A\&A 245, 611

Omont, A. 1990, in Chemistry in Space, Eds. Greenberg and Pironello, p171

Omont, A., Morris, M., Lucas, R., Guilloteau, S. 1991, in preparation

Sahai, R. 1990, ApJ 362, 652

Truong-Bach, Morris, D., Nguyen-Q.-Rieu 1990, A\&A 230, 431

Truong-Bach, Morris, D., Nguyen-Q.-Rieu 1991, A\&A, to be published

Tsuji, T. 1987, in Astrochemistry, IAU Symp 120

Turner, B.E., preprint

Ukita, N., Morris, M. 1983, A\&A 121, L5 


\section{QUESTIONS AND ANSWERS}

B.Khare: Larger molecules are found in the outer envelopes. How large are these molecules?

R.Lucas: $H C_{11} N$ is the largest one.

L.F.Rodriguez: Your HCN results on VY CMA show that the source is very elongated. Do you have an explanation for that?

R.Lucas: Not at this time. 